\title{
Rank reduction and conjugation
}

\author{
Aurél Galántai
}




\title{
RANK REDUCTION AND CONJUGATION
}

\author{
Aurél Galántai \\ Institute of Mathematics, University of Miskolc \\ 3515 Miskolc - Egyetemváros, Hungary \\ matgal@gold.uni-miskolc.hu
}

[Received September 22, 1999]

\begin{abstract}
We investigate Egerváry's rank reduction method and related conjugation algorithms. We give an exact characterization of the full rank factorization produced by the rank reduction algorithm and exploit this result concerning matrix decompositions and conjugation procedures.
\end{abstract}

Mathematical Subject Classification: 65F10,65F30,65F99, 15A22, 15A23

Keywords: Rank reduction, conjugation, matrix factorizations

\section{Introduction}

In the paper we investigate the rank reduction procedure of Egerváry [8], [10], [11], [24] and conjugation algorithms which are related to the following class of conjugate direction methods [25] for solving linear systems of the form

$$
A x=b \quad\left(A \in R^{m \times m}, b \in R^{m}, \operatorname{det}(A) \neq 0\right) .
$$

\section{ALGORITHM 1}

Let $y_{1} \in R^{m}$ be arbitrary.

for $k=1: m$

$$
\begin{aligned}
& r_{k}=A y_{k}-b \\
& \alpha_{k}=v_{k}^{T} r_{k} /\left(v_{k}^{T} A p_{k}\right) \\
& y_{k+1}=y_{k}-\alpha_{k} p_{k}
\end{aligned}
$$

end

Let $P=\left[p_{1}, p_{2}, \ldots, p_{m}\right] \in R^{m \times m}$ and $V=\left[v_{1}, v_{2}, \ldots, v_{m}\right] \in R^{m \times m}$ be nonsingular. The pair $(P, V)$ is said to be $A$-conjugate if the matrix $L=V^{T} A P$ is lower triangular. The pair $(P, V)$ is said to be $A$-biconjugate if $V^{T} A P=D$ is diagonal. Algorithm 1 terminates in $m$ steps if $A y_{m+1}=b$. Combining the results of Stewart [25] and Broyden [4] we can establish the following

Theorem 1 Algorithm 1 terminates in $m$ steps for any starting point $y_{1}$, if and only if the pair $(P, V)$ is A-conjugate.

A particular conjugate direction method is defined by the $A$-conjugate pair $(P, V)$. In the paper we investigate conjugation procedures which are related to the rank 
reduction algorithm of Egerváry. Thus we deal with the $A B S$ conjugation procedure of Abaffy, Broyden and Spedicato [1],[3], the conjugation procedure of Stewart [25], the two-sided Gram-Schmidt procedure of Hegedûs [16], [17], [18], Parlett [21], Chu, Funderlic and Golub [6] (for other $A$-conjugation procedures, see Voyevodin [26]).

It should be mentioned that the rank reduction algorithm was rediscovered concerning the $A B S$ methods which were developed using quasi-Newton techniques [3].

We define $A$-conjugation for rectangular matrices as well. Let $A \in R^{m \times n}, V \in$ $R^{m \times r}$ and $P \in R^{n \times r}$. The pair $(P, V)$ is said to be $A$-conjugate if $L=V^{T} A P$ is nonsingular lower triangular. The pair $(P, V)$ is $A$-biconjugate if $D=V^{T} A P$ is nonsingular diagonal.

Given any matrix $A$, the matrices $A^{\bar{k}}, A^{\mid k}, A^{\underline{k}}$ and $A^{k \mid}$ will denote the submatrices consisting of the first $k$ rows, the first $k$ columns, the last $k$ rows and the last $k$ columns, respectively. Thus $A^{\mid \bar{k}}$ is the leading principal submatrix of order $k$. A nonsingular matrix $A \in R^{m \times m}$ is said to be strongly nonsingular, if $A$ has an $L U$ decomposition. It is well known that a nonsingular matrix $A$ has an $L U$ decomposition, if and only if each $A^{\mid \bar{k}}$ is nonsingular for $k=1, \ldots, m-1$.

In Section 2 we introduce the rank reduction procedure. In Sections 3 and 4 we investigate the necessary and sufficient conditions for performing the rank reduction procedure without breakdown. In Section 5 we give an exact characterization for the components of the full rank factorization $H_{1}=Q_{r} D_{r}^{-1} P_{r}^{T} \in R^{m \times n}$ produced by the rank reduction algorithm and derive various factorization results. It is also shown that the components of the full rank factorization are biconjugate. More precisely the pair $\left(Q_{r}, P_{r}\right)$ is $H_{1}^{-}$-biconjugate, where $H_{1}^{-}$denotes any $g$-inverse of $H_{1}$. The $A B S$ conjugation procedure, which produces $I$-conjugate pairs $(X, P)$, is investigated in Section 6 . Here we show that $A B S$ conjugation is essentially a full rank factorization by Egerváry's rank reduction algorithm. The equivalence of Stewart's conjugation algorithm and the $A B S$ conjugation is shown in Section 7. In Section 8 we investigate the TSGS biconjugation process of Hegedûs [16], [17], [18], Parlett [21], Chu, Funderlic and Golub [6]. It is shown to be a special case of Egerváry's rank reduction algorithm. Section 9 reviews some of the TSGS related results of Chu, Funderlic and Golub [6] giving new proofs as well.

\section{Egerváry's rank reduction procedure}

The rank reduction procedure is based on the following theorem of Egerváry [10] and Wedderburn [28].

Theorem 2 (Egerváry [10]) Let $A \in R^{m \times n}$ be arbitrary with $\operatorname{rank}(A) \geq 1$. Then

$$
\operatorname{rank}\left(A-b c^{T}\right)=\operatorname{rank}(A)-1 \quad\left(b \in R^{m}, c \in R^{n}\right)
$$

holds if and only if $b c^{T}=A u v^{T} A / v^{T} A u$, where $u \in R^{m}, v \in R^{n}$ are arbitrary vectors subject only to the restriction $v^{T} A u \neq 0$. 
It was Wedderburn who proved the if part of the theorem first [28]. Therefore we call this result the Egerváry-Wedderburn theorem. For other proofs and related matters we refer to Elsner-Rózsa [12] and Ouellette [20]. It is also noted that some authors mistakenly attribute Theorem 2 only to Wedderburn ([19]), to Householder ([20]) or to Wedderburn and Householder ([7]).

The rank reduction procedure of Egerváry [10], [11] is the following.

Let $H_{1} \in R^{m \times n}$ be an arbitrary matrix of rank $r$ and let

$$
H_{k+1}=H_{k}-H_{k} x_{k} y_{k}^{T} H_{k} / y_{k}^{T} H_{k} x_{k}, \quad k=1, \ldots, r
$$

for any vectors $x_{k} \in R^{n}$ and $y_{k} \in R^{m}$ for which $y_{k}^{T} H_{k} x_{k} \neq 0$.

As $\operatorname{rank}\left(H_{k+1}\right)=\operatorname{rank}\left(H_{k}\right)-1$ the procedure terminates in $r$ steps, that is $H_{r+1}=0$. Using the notation $D_{k}=\operatorname{diag}\left(y_{1}^{T} H_{1} x_{1}, \ldots, y_{k}^{T} H_{k} x_{k}\right)$ we can write $H_{k}$ and $H_{1}$ in outer-product form

$$
H_{k+1}=H_{1}-\left[H_{1} x_{1}, \ldots, H_{k} x_{k}\right] D_{k}^{-1}\left[\begin{array}{c}
y_{1}^{T} H_{1} \\
\vdots \\
y_{k}^{T} H_{k}
\end{array}\right]
$$

and

$$
H_{1}=\left[H_{1} x_{1}, \ldots, H_{r} x_{r}\right] D_{r}^{-1}\left[\begin{array}{c}
y_{1}^{T} H_{1} \\
\vdots \\
y_{r}^{T} H_{r}
\end{array}\right],
$$

respectively. It follows from recursion (2.2) that Range $\left(H_{k+1}\right) \subset \operatorname{Range}\left(H_{k}\right)$ and Range $\left(H_{k+1}^{T}\right) \subset$ Range $\left(H_{k}^{T}\right)$. If $X=\left[x_{1}, \ldots, x_{r}\right]$ and $Y=\left[y_{1}, \ldots, y_{r}\right]$, then for $m=n=r$

$$
\operatorname{Null}\left(H_{k}\right)=\operatorname{Span}\left(X^{\mid k-1}\right), \quad \operatorname{Range}\left(H_{k}\right)=\operatorname{Span}^{\perp}\left(Y^{\mid k-1}\right) .
$$

\section{The canonical form of Egerváry}

We seek for conditions under which $k$ steps of successive simple rank reduction operations (2.2) can be performed. We need the following result of Guttman [15] (see also Ouellette [20]).

Lemma 3 (Guttman [15]) Let $A \in R^{m \times n}$ be partitioned in the form

$$
A=\left[\begin{array}{ll}
E & F \\
G & H
\end{array}\right] \text {, }
$$

where $E \in R^{k \times k}$ is nonsingular. Then

$$
\operatorname{rank}(A)=\operatorname{rank}(E)+\operatorname{rank}\left(H-G E^{-1} F\right) .
$$


If both $A$ and $E$ are nonsingular, then the Schur complement $H-G E^{-1} F$ is also nonsingular. The if part of the following basic theorem was first proved by Egerváry [10], [11], while the only if part was first proved by Abaffy, Broyden and Spedicato $[1],[3]$.

Theorem 4 The first $k$ steps of rank reduction algorithm (2.2) can be carried out if and only if $Y^{\mid k T} H_{1} X^{\mid k}$ is strongly nonsingular. If $Y^{\mid k T} H_{1} X^{\mid k}$ is strongly nonsingular, then

$$
H_{k+1}=H_{1}-H_{1} X^{\mid k}\left(Y^{\mid k T} H_{1} X^{\mid k}\right)^{-1} Y^{\mid k T} H_{1}
$$

Proof. We first assume that $k$ successive steps were performed, which means that $y_{i}^{T} H_{i} x_{i} \neq 0$ for $i=1, \ldots, k$. Let $Q_{k}=\left[H_{1} x_{1}, \ldots, H_{k} x_{k}\right]$ and $P_{k}=\left[H_{1}^{T} y_{1}, \ldots H_{k}^{T} y_{k}\right]$. Then

$$
H_{k+1}=H_{1}-Q_{k} D_{k}^{-1} P_{k}^{T}
$$

Let us observe that $Y^{\mid k T} Q_{k}=\left[y_{i}^{T} H_{j} x_{j}\right]_{i, j=1}^{k}=L_{k}=\tilde{L}_{k} D_{k}$ is nonsingular lower triangular and $P_{k}^{T} X^{\mid k}=\left[y_{i}^{T} H_{i} x_{j}\right]_{i, j=1}^{k}=U_{k}=D_{k} \tilde{U}_{k}$ is nonsingular upper triangular. Here $\tilde{L}_{k}$ and $\tilde{U}_{k}$ are unit lower and upper triangular, respectively. We can also observe that

$$
H_{1} X^{\mid k}=\left(H_{1}-H_{k+1}\right) X^{\mid k}=Q_{k} D_{k}^{-1} P_{k}^{T} X^{\mid k}=Q_{k} D_{k}^{-1} U_{k}=Q_{k} \tilde{U}_{k}
$$

from which $Q_{k}=H_{1} X^{\mid k} U_{k}^{-1} D_{k}=H_{1} X^{\mid k} \tilde{U}_{k}^{-1}$ follows. Similarly we obtain that

$$
Y^{\mid k T} H_{1}=Y^{\mid k T}\left(H_{1}-H_{k+1}\right)=Y^{\mid k T} Q_{k} D_{k}^{-1} P_{k}^{T}=L_{k} D_{k}^{-1} P_{k}^{T}=\tilde{L}_{k} P_{k}^{T}
$$

and $P_{k}^{T}=D_{k} L_{k}^{-1} Y^{\mid k T} H_{1}=\tilde{L}_{k}^{-1} Y^{\mid k T} H_{1}$. Hence

$$
H_{k+1}=H_{1}-H_{1} X^{\mid k} \tilde{U}_{k}^{-1} D_{k}^{-1} \tilde{L}_{k}^{-1} Y^{\mid k T} H_{1}=H_{1}-H_{1} X^{\mid k}\left(\tilde{L}_{k} D_{k} \tilde{U}_{k}\right)^{-1} Y^{\mid k T} H_{1} .
$$

As

$$
Y^{\mid k T} H_{1} X^{\mid k}=Y^{\mid k T} Q_{k} D_{k}^{-1} P_{k}^{T} X^{\mid k}=\tilde{L}_{k} D_{k} \tilde{U}_{k}
$$

we proved that $Y^{\mid k T} H_{1} X^{\mid k}$ is strongly nonsingular. By substitution we obtain formula (3.3). Let us suppose that $Y^{\mid k T} H_{1} X^{\mid k}$ is strongly nonsingular. We must prove that $y_{i}^{T} H_{i} x_{i} \neq 0$ for $i=1, \ldots, k$. As by assumption $y_{1}^{T} H_{1} x_{1} \neq 0$ the matrix $H_{2}=$ $H_{1}-H_{1} x_{1} y_{1}^{T} H_{1} / y_{1}^{T} H_{1} x_{1}$ is defined. Let us assume that we have defined $H_{i}$ for $i \leq k$. Then

$$
H_{i}=H_{1}-H_{1}\left(X^{\mid i-1}\right)^{T}\left(\left(Y^{\mid i-1}\right)^{T} H_{1} X^{\mid i-1}\right)^{-1}\left(Y^{\mid i-1}\right)^{T} H_{1}
$$


and

$$
y_{i}^{T} H_{i} x_{i}=y_{i}^{T} H_{1} x_{i}-y_{i}^{T} H_{1}\left(X^{\mid i-1}\right)^{T}\left(\left(Y^{\mid i-1}\right)^{T} H_{1} X^{\mid i-1}\right)^{-1}\left(Y^{\mid i-1}\right)^{T} H_{1} x_{i} .
$$

Let us observe that $y_{i}^{T} H_{i} x_{i}$ is the Schur complement of the bordered matrix

$$
Y^{\mid i T} H_{1} X^{\mid i}=\left[\begin{array}{cc}
\left(Y^{\mid i-1}\right)^{T} H_{1} X^{\mid i-1} & \left(Y^{\mid i-1}\right)^{T} H_{1} x_{i} \\
y_{i}^{T} H_{1} X^{\mid i-1} & y_{i}^{T} H_{1} x_{i}
\end{array}\right]
$$

As $\operatorname{rank}\left(Y^{\mid i T} H_{1} X^{\mid i}\right)=i$ and $\operatorname{rank}\left(\left(Y^{\mid i-1}\right)^{T} H_{1} X^{\mid i-1}\right)=i-1$, the Guttman lemma implies that $\operatorname{rank}\left(y_{i}^{T} H_{i} x_{i}\right)=1$ and $y_{i}^{T} H_{i} x_{i} \neq 0$. Hence $H_{i+1}$ is defined and we completed the proof of the theorem.

The first part of the proof is a slight modification of Theorems 4.2 and 4.3 in [3]. The second part of the proof is new.

We recall that for general bordered matrices of the form

$$
B^{\prime}=\left[\begin{array}{cc}
B & z \\
w^{T} & \lambda
\end{array}\right] \quad\left(B \in R^{k \times k}, z, w \in R^{k}\right)
$$

the identity $\operatorname{det}\left(B^{\prime}\right)=\operatorname{det}(B)\left(\lambda-w^{T} B^{-1} z\right)$ holds, if $B$ is nonsingular. For $i>1$ we can write

$$
y_{i}^{T} H_{i} x_{i}=\operatorname{det}\left(Y^{\mid i T} H_{1} X^{\mid i}\right) / \operatorname{det}\left(\left(Y^{\mid i-1}\right)^{T} H_{1} X^{\mid i-1}\right) .
$$

For $i>1$ it easily follows that

$$
\prod_{j=1}^{i} y_{j}^{T} H_{j} x_{j}=\operatorname{det}\left(Y^{\mid i T} H_{1} X^{\mid i}\right)
$$

This formula is well-known concerning the bordering inversion method, where $1 /(\lambda-$ $w^{T} B^{-1} z$ ) appears in place of $\lambda$ in the inverse of $B^{\prime}$ (see, e.g. [23], [6]).

Using the canonical form (3.3) we can easily prove

Proposition 5 Let $H_{1}^{-}$be any $g$-inverse of $H_{1}$. Then

$$
H_{k} H_{1}^{-} H_{j}=H_{j} H_{1}^{-} H_{k}=H_{k} \quad(j \leq k) .
$$

This result was first observed by Abaffy, Broyden and Spedicato for nonsingular $H_{1}$ [1], [3]. Let us notice that $H_{1}^{-} H_{k}$ 's are projectors. Particularly, for $H_{1}=I \in R^{m \times m}$ all $H_{k}$ 's are projectors.

For $k=r$ the canonical form (3.3) leads to the decomposition

$$
H_{1}=H_{1} X\left(Y^{T} H_{1} X\right)^{-1} Y^{T} H_{1},
$$


where $Z=X\left(Y^{T} H_{1} X\right)^{-1} Y^{T}$ satisfies $H_{1} Z H_{1}=H_{1}$ and $Z H_{1} Z=Z$. Hence $Z$ is a reflexive $g$-inverse of $H_{1}$. If $H_{1}=F G$ is a full rank factorization, $X=G^{T} M$ and $Y=F N\left(M, N \in R^{r \times r}\right.$ any nonsingular matrices), then $Z=X\left(Y^{T} H_{1} X\right)^{-1} Y^{T}$ is the Moore-Penrose inverse of $H_{1}$. For other properties of the reflexive $g$-inverse $Z$ we refer to Egerváry [9].

Proposition 6 If $H_{1}=F G$ is a full rank factorization, $X=G^{T}\left(G G^{T}\right)^{-1}$ and $Y=F\left(F^{T} F\right)^{-1}$, then $H_{k+1}$ can be factorized in the form

$$
H_{k+1}=F\left(I-I^{\mid k} I^{\bar{k}}\right) G \quad\left(I \in R^{r \times r}\right) .
$$

\section{Block rank reduction}

Egerváry considered formula (3.3) as one block rank reduction [10], [11]. Block rank reduction was also investigated by Cline and Funderlic [7], and Ouellette [20] (see also Rao [22]). Here we recall the result of Cline and Funderlic ([7], Corollary 3.1) and give a new proof which exploits a technique of Ouellette ([20], Thms. 2.6a and 2.6b).

Theorem 7 (Cline-Funderlic [7]) Let $H, S \in R^{m \times n}, \operatorname{rank}(H) \geq \operatorname{rank}(S)=k$ and let $U R^{-1} V^{T}\left(U \in R^{m \times k}, R \in R^{k \times k}, V \in R^{n \times k}\right)$ be a full rank factorization of $S$. Then

$$
\operatorname{rank}(H-S)=\operatorname{rank}(H)-\operatorname{rank}(S)
$$

if and only if

$$
U=H X, \quad V^{T}=Y^{T} H, \quad Y^{T} H X=R
$$

for some matrices $X \in R^{n \times k}$ and $Y \in R^{m \times k}$.

Proof. We first prove the if part. Let us consider the matrix

$$
B=\left[\begin{array}{cc}
Y^{T} H X & Y^{T} H \\
H X & H
\end{array}\right]=\left[\begin{array}{c}
Y^{T} \\
I_{m}
\end{array}\right] H\left[X, I_{n}\right] .
$$

From the Sylvester law of nullity it follows that $\operatorname{rank}(H)=\operatorname{rank}(B)$. Applying the Guttman lemma we obtain

$$
\operatorname{rank}\left(H-H X\left(Y^{T} H X\right)^{-1} Y^{T} H\right)=\operatorname{rank}(H)-\operatorname{rank}(S) .
$$

The only if part is demonstrated in the following way. Let us assume that $\operatorname{rank}(H-S)<$ $\operatorname{rank}(H)-k+1$ and let

$$
B=\left[\begin{array}{cc}
R & V^{T} \\
U & H
\end{array}\right]
$$


As by the Guttman lemma

$$
\operatorname{rank}(H) \leq \operatorname{rank}(B)=\operatorname{rank}(R)+\operatorname{rank}\left(H-U R^{-1} V^{T}\right)<\operatorname{rank}(H)+1,
$$

we obtain that $\operatorname{rank}(H)=\operatorname{rank}(B)$. Hence there exist matrices $X$ and $Y$ such that

$$
\left[R, V^{T}\right]=Y^{T}[U, H], \quad\left[\begin{array}{c}
R \\
U
\end{array}\right]=\left[\begin{array}{c}
V^{T} \\
H
\end{array}\right] X .
$$

From here we obtain that $V^{T}=Y^{T} H, U=H X$ and $R=V^{T} X=Y^{T} U=Y^{T} H X$. Thus we proved the theorem.

Remark 8 The case $k=1$ gives the Wedderburn-Egerváry theorem. The if part of Theorem 7 was first proved by Egerváry [10], [11] and also appears in Rao [22]. Ouellette investigated the block rank reduction

$$
H-H X\left(Y^{T} H X\right)^{-} Y^{T} H
$$

with rectangular $Y^{T} H X$ and any choice of generalized inverse [20].

The block rank reduction and the successive simple rank reductions are not equivalent. Egerváry proved that for strongly nonsingular $Y^{\mid k T} H_{1} X^{\mid k}$ the block reduction (3.3) is obtainable by $k$ successive simple rank operations [10], [11]. This is not the case if $Y^{\mid k T} H_{1} X^{\mid k}$ is not strongly nonsingular as shown by the following example

$$
H_{1}=\left[\begin{array}{lll}
0 & 1 & 0 \\
1 & 1 & 0 \\
0 & 0 & 1
\end{array}\right], \quad X=Y=I, \quad k=2 .
$$

So the conditions of block rank reduction are less restrictive. We can easily extend the canonical formula of Egerváry to the block rank reduction algorithm

$$
H_{k+1}=H_{k}-H_{k} X_{k}\left(Y_{k}^{T} H_{k} X_{k}\right)^{-1} Y_{k}^{T} H_{k}, \quad k=1, \ldots, t,
$$

where $X_{k} \in R^{n \times l_{k}}, Y_{k} \in R^{m \times l_{k}}, l_{k} \geq 1$ and $\operatorname{rank}\left(H_{1}\right) \geq \sum_{k=1}^{t} l_{k}$.

Let $m=\sum_{i=1}^{k} l_{i}$. A matrix $A \in R^{m \times m}$ is said to be partitioned with respect to the partition $\left\{l_{1}, \ldots, l_{k}\right\}$, if $A=\left[A_{i j}\right]_{i, j=1}^{k}$ and $A_{i j} \in R^{l_{i} \times l_{j}}$. A matrix $A$ is said to be block strongly nonsingular with respect to the partition $\left\{l_{1}, \ldots, l_{k}\right\}$ if there exist nonsingular block lower triangular $L$ and nonsingular block upper triangular $U$ such that $A=L U$, and both matrices are partitioned with respect to $\left\{l_{1}, \ldots, l_{k}\right\}$. Let $\tilde{X}^{\mid k}=\left[X_{1}, \ldots, X_{k}\right], \widetilde{Y}^{\mid k}=\left[Y_{1}, \ldots, Y_{k}\right]$. Then

$$
\operatorname{Range}\left(H_{k}\right)=\operatorname{Span}^{\perp}\left(\tilde{Y}^{\mid k-1}\right), \quad \operatorname{Null}\left(H_{k}\right)=\operatorname{Span}\left(\tilde{X}^{\mid k-1}\right) .
$$

The following result can be proved in the same way as Theorem 4 . 
Theorem 9 The first $k$ steps of block rank reduction algorithm (4.3) can be carried out if and only if $\widetilde{Y}^{\mid k T} H_{1} \widetilde{X}^{\mid k}$ is block strongly nonsingular. If $\widetilde{Y}^{\mid k T} H_{1} \widetilde{X}^{\mid k}$ is block strongly nonsingular, then

$$
H_{k+1}=H_{1}-H_{1} \tilde{X}^{\mid k}\left(\widetilde{Y}^{\mid k T} H_{1} \tilde{X}^{\mid k}\right)^{-1} \tilde{Y}^{\mid k T} H_{1} .
$$

The only if part of the theorem first appeared in [2] with a slightly different proof (see, also [3]). If $\widetilde{Y}^{\mid k T} H_{1} \widetilde{X}^{\mid k}$ is strongly nonsingular in the usual sense, then it is also block strongly nonsingular for any partition. In this case the if part of the theorem follows from Theorem 4, which is a special case of Theorem 9. We also note that all the results of subsequent sections which follow from Theorem 4 can also be established for the block case in a straightforward way.

\section{Matrix factorizations and rank reduction}

We determine the components of the full rank factorization (2.4) in terms of the parameters $H_{1}, X$ and $Y$ and derive important consequences such as matrix decompositions, orthogonalization and similarity transformations to Hessenberg and tridiagonal forms. It is also shown that the pair $\left(Q_{r}, P_{r}\right)$ is $H_{1}^{-}$-biconjugate, where $H_{1}^{-}$is any $g$-inverse.

Let $B=L_{B} D_{B} U_{B}$ be the unique $L D U$-decomposition of matrix $B \in R^{m \times m}$ with unit lower triangular $L_{B}$, diagonal $D_{B}$ and unit upper triangular $U_{B}$. We later use the following simple observations.

If $Y$ is nonsingular lower triangular, then $L_{Y A}=Y L_{A} D_{Y}^{-1}, D_{Y A}=D_{Y} D_{A}$ and $U_{Y A}=U_{A}$. If $X$ is nonsingular upper triangular, then $L_{A X}=L_{A}, D_{A X}=D_{A} D_{X}$ and $U_{A X}=D_{X}^{-1} U_{A} X$. Let $Y$ be a nonsingular lower triangular matrix. $A$ is strongly nonsingular, if and only if $Y A$ is also strongly nonsingular. Similarly, let $X$ be a nonsingular upper triangular matrix. Then $A$ is strongly nonsingular if and only if $A X$ is also strongly nonsingular.

If two nonsingular matrices $A$ and $B$ have the $L U$-decompositions $A=L_{1} U$ and $B=L_{2} U$ with the same upper triangular $U$, then there is a unique lower triangular matrix $L$ such that $A=L B$. Similarly, if $A=L U_{1}$ and $B=L U_{2}$ hold with the same lower triangular $L$, then there is a unique upper triangular matrix $U$ such that $A=B U$.

From the proof of Theorem 4 (formula (3.4)) we can easily establish that

$$
\begin{gathered}
\tilde{L}_{k}=L_{Y^{\mid k T} H_{1} X^{\mid k}}, \quad D_{k}=D_{Y^{\mid k T} H_{1} X^{\mid k}}, \quad \widetilde{U}_{k}=U_{Y^{\mid k T} H_{1} X^{\mid k}}, \\
P_{k}^{T}=L_{Y^{\mid k T} H_{1} X^{\mid k}}^{-1} Y^{\mid k T} H_{1}, \quad Q_{k}=H_{1} X^{\mid k} U_{Y^{\mid k T} H_{1} X^{\mid k}}^{-1} .
\end{gathered}
$$

For $k=r$ we obtain the components of Egerváry's full rank factorization in terms of the parameters $X, Y$ and $H_{1}$. 
Theorem 10 Let $H_{1} \in R^{m \times n}$ of rank $r$ and let $Y^{T} H_{1} X$ be strongly nonsingular. The components of the full rank factorization $H_{1}=Q_{r} D_{r}^{-1} P_{r}^{T}$ are

$$
P_{r}=H_{1}^{T} Y L_{Y^{T} H_{1} X}^{-T}, \quad Q_{r}=H_{1} X U_{Y^{T} H_{1} X}^{-1}, \quad D_{r}=D_{Y^{T} H_{1} X},
$$

and $H_{1}$ can be expressed in the form

$$
H_{1}=\left(H_{1} X U_{Y^{T} H_{1} X}^{-1}\right) D_{Y^{T} H_{1} X}^{-1}\left(L_{Y^{T} H_{1} X}^{-1} Y^{T} H_{1}\right) .
$$

Expression (5.3) for $P_{r}$ first appeared in [13], [14] with a different proof. It was not realized until writing this paper that the proof of Theorem 4 can be modified so that expressions (5.3)-(5.4) could be derived easily.

The matrices $P_{r}$ and $Q_{r}$ can be written in the form $P_{r}=H_{1}^{T} Y U_{X^{T} H_{1}^{T} Y}^{-1}$ and $Q_{r}=$ $H_{1} X L_{X^{T} H_{1}^{T} Y}^{-T}$, respectively. The role of $P_{r}$ can be changed with $Q_{r}$ by transposing $H_{1}$. As

$$
H_{1}^{T}=\left(H_{1}^{T} Y U_{X^{T} H_{1}^{T} Y}^{-1}\right) D_{X^{T} H_{1} Y}^{-1}\left(L_{X^{T} H_{1}^{T} Y^{-1}}^{-T} H_{1}^{T}\right)
$$

several results on $P_{r}$ can be formulated for $Q_{r}$ if we replace $H_{1}$ by $H_{1}^{T}, X$ and $Y$ by each other.

We also note that for nonsingular upper triangular $U_{1}$ and $U_{2}$ the transformations $Y \rightarrow Y U_{1}$ and $X \rightarrow X U_{2}$ change the factorization (5.4) to

$$
H_{1}=\left(H_{1} X U_{Y^{T} H_{1} X}^{-1} D_{U_{2}}\right)\left(D_{U_{2}}^{-1} D_{Y^{T} H_{1} X}^{-1} D_{U_{1}}^{-1}\right)\left(D_{U_{1}} L_{Y^{T} H_{1} X}^{-1} Y^{T} H_{1}\right) .
$$

Hence the effect of these transformations is only a diagonal scaling.

The following observation enlightens a basic property of the full rank factorization (5.4).

Proposition 11 Let $H_{1}^{-}$denote any g-inverse of $H_{1}$. Then the pair $\left(Q_{r}, P_{r}\right)$ is $H_{1}^{-}$biconjugate.

Proof. By definition

$$
P_{r}^{T} H_{1}^{-} Q_{r}=L_{Y^{T} H_{1} X}^{-1} Y^{T}\left(H_{1} H_{1}^{-} H_{1}\right) X U_{Y^{T} H_{1} X}^{-1}=D_{Y^{T} H_{1} X} .
$$

The rank reduction procedure defines a full rank factorization (5.4) of matrix $H_{1}$. The next observation, which follows from Theorem 10, shows that all full rank factorizations can be generated by Egerváry's rank reduction procedure.

Proposition 12 Let $H_{1} \in R^{m \times n}$ be of rank $r$ and let $H_{1}=F G$ be any full rank factorization. Let $X=G^{T}\left(G G^{T}\right)^{-1}$ and $Y=F\left(F^{T} F\right)^{-1}$. Then $Q_{r}=F, D_{r}=I$ and $P_{r}^{T}=G$. 
We mention here two simple examples.

Example 13 Let the singular value decomposition of $H_{1} \in R^{m \times n}$ be given in the form $H_{1}=U\left(\Sigma V^{T}\right)$, where $U \in R^{m \times r}, V \in R^{m \times r}, U^{T} U=I, V^{T} V=I$ and $\Sigma \in R^{r \times r}$ is nonsingular. The choice $F=U, G=\Sigma V^{T}$ yields $X=V \Sigma^{-1}$ and $Y=U$. Thus $Q_{r}=U$ and $P_{r}=\Sigma V^{T}$.

The polar decomposition theorem says that every nonsingular matrix $A \in R^{m \times m}$ can be written in the form $A=Q P$, where $Q$ is an orthogonal and $P$ is a symmetric positive definite matrix.

Example 14 Let $H_{1} \in R^{m \times m}$ be nonsingular and let $H_{1}=Q P$ be its polar decomposition. The choice $F=P, G=Q$ yields $X=P^{-1}$ and $Y=Q$. Thus $Q_{m}=Q$ and $P_{m}^{T}=P$.

We consider now the matrix factorizations we can obtain from Theorem 10. For the rest of this section we assume that all matrices $H_{1}, X, Y \in R^{m \times m}$ are nonsingular so that $X^{T} H_{1} Y$ are strongly nonsingular. Then $Q_{m}$ and $P_{m}$ are also nonsingular, $Q_{m}=H_{1} P_{m}^{-T} D_{m}$ and formula (5.4) can be written in the form

$$
H_{1}=\left(Y^{-T} L_{Y^{T} H_{1} X} D_{Y^{T} H_{1} X}\right) D_{Y^{T} H_{1} X}^{-1}\left(D_{Y^{T} H_{1} X} U_{Y^{T} H_{1} X} X^{-1}\right) .
$$

Proposition $15 P_{m}^{T}$ is upper triangular if and only if $X$ is upper triangular. $P_{m}^{T}$ is lower triangular if and only if $Y^{T} H_{1}$ is lower triangular. $Q_{m}$ is upper triangular if and only if $H_{1} X$ is upper triangular. $Q_{m}$ is lower triangular if and only if $Y^{T}$ is lower triangular.

Proof. From the relation $P_{m}^{T}=\left(D_{Y^{T} H_{1} X} U_{Y^{T} H_{1} X}\right) X^{-1}=\widetilde{U}$, where $\widetilde{U}$ is upper triangular, it follows that $X^{-1}=U_{Y^{T} H_{1} X}^{-1} D_{Y^{T} H_{1} X}^{-1} \widetilde{U}$ is also upper triangular. Hence $X$ is upper triangular. If $P_{m}^{T}=L_{Y^{T} H_{1} X}^{-1} Y^{T} H_{1}=\widetilde{L}$, where $\widetilde{L}$ is lower triangular, then $Y^{T} H_{1}=L_{Y^{T} H_{1} X} \widetilde{L}$ is also lower triangular. Similarly, $Q_{m}=H_{1} X U_{Y^{T} H_{1} X}^{-1}=\widetilde{U}$, if and only if $H_{1} X$ is upper triangular. Finally, $Q_{m}=Y^{-T} L_{Y^{T} H_{1} X} D_{Y^{T} H_{1} X}=\widetilde{L}$, if and only if $Y^{T}$ is lower triangular.

Corollary $16 Q_{m}$ is lower triangular and $P_{m}^{T}$ is upper triangular, if and only if $X$ and $Y$ are upper triangular.

In this case factorization (5.4) simplifies to the $L U$ factorization

$$
H_{1}=\left(L_{H_{1}} D_{H_{1}} D_{X}\right)\left(D_{X}^{-1} D_{H_{1}}^{-1} D_{Y^{T}}^{-1}\right)\left(D_{Y^{T}} D_{H_{1}} U_{H_{1}}\right)
$$

in agreement with (5.6). For $X=Y=I$ this result was first observed by Egerváry [8], [10] (for $A B S$ conjugation, see [3]). 
Proposition $17 P_{m}$ is upper Hessenberg if and only if $H_{1}^{T} Y$ is upper Hessenberg. $Q_{m}$ is upper Hessenberg if and only if $H_{1} X$ is upper Hessenberg.

Proof. We exploit the fact that the upper Hessenberg form is invariant under multiplication by upper triangular matrix from both sides. By definition $P_{m}=$ $H_{1}^{T} Y L_{Y^{T} H_{1} X}^{-T}=F$, where $F$ is upper Hessenberg if and only if $H_{1}^{T} Y=F L_{Y^{T} H_{1} X}^{T}$ is upper Hessenberg. Similarly, $Q_{m}=H_{1} X U_{Y^{T} H_{1} X}^{-1}=F$ is upper Hessenberg if and only if $H_{1} X=F U_{Y^{T} H_{1} X}$ is upper Hessenberg.

Proposition 18 Let $B \in R^{m \times m}$ be a symmetric and positive definite matrix. $P_{m}$ is $B$-orthogonal if and only if $X=B H_{1}^{T} Y U$ holds with any nonsingular $Y$ and $a$ nonsingular upper triangular $U$.

Proof. $P_{m}$ is $B$-orthogonal, if and only if

$$
P_{m}^{T} B P_{m}=L_{Y^{T} H_{1} X}^{-1} Y^{T} H_{1} B H_{1}^{T} Y L_{Y^{T} H_{1} X}^{-T}=D,
$$

where $D$ is diagonal. This holds exactly if $Y^{T} H_{1} B H_{1}^{T} Y=L_{Y^{T} H_{1} X} D L_{Y^{T} H_{1} X}^{T}$. This implies $L_{Y^{T} H_{1} B H_{1}^{T} Y}=L_{Y^{T} H_{1} X}$. Hence a nonsingular upper triangular $U$ exists such that $Y^{T} H_{1} B H_{1}^{T} Y U=Y^{T} H_{1} X$, from which $X=B H_{1}^{T} Y U$ follows. As $B$ is symmetric and positive definite, the nonsingularity of $Y$ and $U$ is the condition for the strong nonsingularity of $Y^{T} H_{1} X$.

We can drop the positive definiteness condition of $B$ if we assume that $Y^{T} H_{1} X=$ $Y^{T} H_{1} B H_{1}^{T} Y$ is strongly nonsingular.

Corollary $19 P_{m}$ is orthogonal up to a diagonal scaling and $Q_{m}$ is lower triangular if and only if $X=H_{1}^{T} Y U$ holds with any nonsingular upper triangular $U$ and $Y$. In such a case, factorization (5.4) is the LQ-factorization of $H_{1}$.

Proposition 20 Let $B \in R^{m \times m}$ be a symmetric and positive definite matrix. $Q_{m}$ is B-orthogonal, if and only if $Y=B H_{1} X L^{T}$ holds with any nonsingular $X$ and $a$ nonsingular lower triangular $L$.

Proof. $Q_{m}$ is $B$-orthogonal if and only if

$$
Q_{m}^{T} B Q_{m}=U_{Y^{T} H_{1} X}^{-T} X^{T} H_{1}^{T} B H_{1} X U_{Y^{T} H_{1} X}^{-1}=D
$$

where $D$ is diagonal. This holds exactly if $X^{T} H_{1}^{T} B H_{1} X=U_{Y^{T} H_{1} X}^{T} D U_{Y^{T} H_{1} X}$ from which $U_{X^{T} H_{1}^{T} B H_{1} X}=U_{Y^{T} H_{1} X}$ follows. Hence a nonsingular lower triangular matrix $L$ exists such that $L X^{T} H_{1}^{T} B H_{1} X=Y^{T} H_{1} X$. This implies $Y=B H_{1} X L^{T}$. As $B$ is symmetric and positive definite, the nonsingularity of $X$ and $L$ is the condition for the strong nonsingularity of $Y^{T} H_{1} X$.

Corollary $21 Q_{m}$ is orthogonal up to a diagonal scaling and $P_{m}^{T}$ is upper triangular if and only if $Y=H_{1} X L^{T}$ holds with any nonsingular lower triangular $L$ and upper triangular $X$. In such a case, factorization (5.4) is the QR-factorization of $H_{1}$. 

[3].

We note that special cases of Corollaries 19 and 21 appear in the $A B S$ conjugation

Given a matrix $H_{1} \in R^{m \times m}$, a $G R$ decomposition of $H_{1}$ is a factorization of $H_{1}$ into a product $B=G R$, where $G$ is nonsingular and $R$ is upper triangular [27]. By Proposition 12 any $G R$ decomposition can be obtained by the rank reduction algorithm of Egerváry. For special cases such as the $L U$ and $Q R$ factorizations we refer to Corollaries 16 and 21.

Finally we investigate the special case $H_{1}=I$. Then

$$
I=\left(X U_{Y^{T} X}^{-1}\right) D_{Y^{T} X}^{-1}\left(L_{Y^{T} X}^{-1} Y^{T}\right)
$$

and the factors $Q_{m}=X U_{Y^{T} X}^{-1}=Y^{-T} L_{Y^{T} X} D_{Y^{T} X}, D_{m}=D_{Y^{T} X}$ and $P_{m}=Y L_{Y^{T} X}^{-T}=$ $X^{-T} U_{Y^{T} X}^{T} D_{Y^{T} X}$ are such that $Q_{m}^{T}=\left(P_{m} D_{m}^{-1}\right)^{-1}$.

The following table gives some $L U$ factorization related results for $H_{1}=I$ and the parameters given therein.

\begin{tabular}{|l|l|l|l|l|}
\hline$X$ & $Y$ & $Q_{m}$ & $D_{m}^{-1}$ & $P_{m}^{T}$ \\
\hline$A$ & $I$ & $L_{A} D_{A}$ & $D_{A}^{-1}$ & $L_{A}^{-1}$ \\
\hline$A^{T}$ & $I$ & $U_{A}^{T} D_{A}$ & $D_{A}^{-1}$ & $U_{A}^{-T}$ \\
\hline$I$ & $A$ & $L_{A}^{-T}$ & $D_{A}^{-1}$ & $D_{A} L_{A}^{T}$ \\
\hline$I$ & $A^{T}$ & $U_{A}^{-1}$ & $D_{A}^{-1}$ & $D_{A} U_{A}$ \\
\hline
\end{tabular}

It is noted that the parameters $X=A^{T}$ and $Y=I$ define the implicit $L U A B S$ method (see [3]).

The following observations are related to the Lánczos reductions (see, e.g. [19]). Let $y_{1} \in R^{m}$ and $y_{i}=A^{i-1} y_{1}(i=2, \ldots, m)$. Let $y_{1} \in R^{m}$ be such that the Krylov matrix $Y=\left[y_{1}, A y_{1}, \ldots, A^{m-1} y_{1}\right]$ is nonsingular. Then $A^{m} y=\sum_{i=1}^{m} \gamma_{i} A^{i-1} y_{1}$ and $A Y=Y F$, where the companion matrix $F$ is defined by

$$
F=\left[\begin{array}{ccccc}
0 & 0 & \ldots & 0 & \gamma_{1} \\
1 & 0 & & \vdots & \vdots \\
0 & 1 & \ddots & \vdots & \vdots \\
\vdots & \ddots & \ddots & 0 & \gamma_{m-1} \\
0 & \ldots & 0 & 1 & \gamma_{m}
\end{array}\right]
$$

We exploit that $Q_{m}^{T}=\left(P_{m} D_{m}^{-1}\right)^{-1}$ for $H_{1}=I$. Let us consider the similarity transformation

$$
\begin{aligned}
Q_{m}^{T} A\left(P_{m} D_{m}^{-1}\right) & =D_{Y^{T} X} L_{Y^{T} X}^{T}\left(Y^{-1} A Y\right) L_{Y^{T} X}^{-T} D_{Y^{T} X}^{-1} \\
& =D_{Y^{T} X} L_{Y^{T} X}^{T} F L_{Y^{T} X}^{-T} D_{Y^{T} X}^{-1},
\end{aligned}
$$

where the upper Hessenberg matrix $F$ is multiplied by two upper triangular matrices. This multiplication keeps the upper Hessenberg form. Hence Egerváry's 
rank reduction algorithm defines a similarity transformation of matrix $A$ to upper Hessenberg form and gives the transformation matrices $Q_{m}^{T}$ and $Q_{m}^{-T}$ simultaneously. Let us assume again that $x_{1} \in R^{m}$ is such that the Krylov matrix $X=\left[x_{1}, A^{T} x_{1}, \ldots,\left(A^{T}\right)^{m-1} x_{1}\right]$ is nonsingular. Then $A^{T} X=X F_{1}$ holds with a suitable companion matrix $F_{1}$. With this choice of $X$, relation (5.9) becomes

$$
Q_{m}^{T} A\left(P_{m} D_{m}^{-1}\right)=U_{Y^{T} X}^{-T}\left(X^{T} A X^{-T}\right) U_{Y^{T} X}^{T}=U_{Y^{T} X}^{-T} F_{1}^{T} U_{Y^{T} X}^{T},
$$

where the lower Hessenberg matrix $F_{1}^{T}$ is multiplied by two lower triangular matrices. This multiplication keeps the lower Hessenberg form. As $Q_{m}^{T} A\left(P_{m} D_{m}^{-1}\right)$ is of both upper and lower Hessenberg form, the matrix must be tridiagonal. Hence the rank reduction algorithm also defines a similarity transformation of matrix $A$ to tridiagonal form giving the transformation matrices $Q_{m}^{T}$ and $Q_{m}^{-T}$ simultaneously.

Theorem 22 Let $y_{1} \in R^{m}$ be such that $Y=\left[y_{1}, A y_{1}, \ldots, A^{m-1} y_{1}\right]$ is nonsingular. If $H_{1}=I$ and $X$ is such that $Y^{T} X$ is strongly nonsingular, then $Q_{m}^{T} A\left(P_{m} D_{m}^{-1}\right)$ defines an upper Hessenberg matrix which is similar to $A$. If, in addition $X=$ $\left[x_{1}, A^{T} x_{1}, \ldots,\left(A^{T}\right)^{m-1} x_{1}\right]$, then $Q_{m}^{T} A\left(P_{m} D_{m}^{-1}\right)$ is a tridiagonal matrix, which is similar to $A$.

Similar results appeared in Stewart [25], and Abaffy and Spedicato [3] in association with the $A$-conjugation algorithms. What is really new here is the simultaneous computation of the transformation matrix and its inverse.

\section{The $A B S$ conjugation procedure}

In this section we investigate the $A B S$ conjugation procedure of Abaffy, Broyden and Spedicato [3]. This algorithm, which is based on the rank reduction procedure, produces $A$-conjugate pairs $(X, P)$. We assume that $H_{1}, A \in R^{m \times m}$ are nonsingular.

\section{ALGORITHM 2}

$H_{1} \in R^{m \times m}$ is arbitrary nonsingular

$$
\begin{aligned}
& \text { for } k=1: m \\
& \quad p_{k}=H_{k}^{T} z_{k} \quad\left(z_{k} \in R^{m}, x_{k}^{T} p_{k} \neq 0\right) \\
& \quad H_{k+1}=H_{k}-H_{k} x_{k} y_{k}^{T} H_{k} / y_{k}^{T} H_{k} x_{k} \quad\left(y_{k} \in R^{m}, y_{k}^{T} H_{k} x_{k} \neq 0\right) \\
& \text { end }
\end{aligned}
$$

As for $i<j$ the relation $x_{i} \in N$ ull $\left(H_{j}\right)$ implies $x_{i}^{T} I p_{j}=x_{i}^{T} H_{j}^{T} p_{j}=0$, the matrix $L=X^{T} P$ is nonsingular lower triangular. Hence Algorithm 2 produces the $I$-conjugate pair $(P, X)$. The substitution $x_{i}=A^{T} v_{i}(i=1, \ldots, m)$ or $X=A^{T} V$ yields $L=X^{T} P=V^{T} A P$. So the resulting pair $(P, V)$ is $A$-conjugate. Therefore it is enough to formulate and prove statements only for $I$-conjugate pairs.

We can also define the conjugate pair $(Q, Y)$ by setting $q_{k}=H_{k} w_{k}(k=1, \ldots, m)$ and $Q=\left[q_{1}, \ldots, q_{m}\right]$. For $i<j, y_{i}^{T} I q_{j}=y_{i}^{T} H_{j} w_{j}=0$. Hence the pair $(Q, Y)$ is indeed $I$-conjugate. 
For further use let $Z=\left[z_{1}, \ldots, z_{m}\right]$. We seek for conditions under which Algorithm 2 produces nonsingular $I$-conjugate pairs $(P, X)$. This happens if and only if

$$
x_{k}^{T} p_{k} \neq 0, \quad y_{k}^{T} H_{k} x_{k} \neq 0 \quad(i=1, \ldots, m) .
$$

The second condition holds if and only if $X^{T} H_{1} Y$ is strongly nonsingular. By definition $x_{1}^{T} p_{1}=x_{1}^{T} H_{1}^{T} z_{1}$ and

$$
x_{k+1}^{T} p_{k+1}=x_{k+1}^{T} H_{1}^{T} z_{k+1}-x_{k+1}^{T} H_{1}^{T} Y^{\mid k}\left(X^{\mid k T} H_{1}^{T} Y^{\mid k}\right)^{-1} X^{\mid k T} H_{1}^{T} z_{k+1},
$$

which is the Schur complement of the bordered matrix

$$
\left(X^{\mid k+1}\right)^{T} H_{1}^{T}\left[Y^{\mid k}, z_{k+1}\right]=\left[\begin{array}{cc}
X^{\mid k T} H_{1}^{T} Y^{\mid k} & X^{\mid k T} H_{1}^{T} z_{k+1} \\
x_{k+1}^{T} H_{1}^{T} Y^{\mid k} & x_{k+1}^{T} H_{1}^{T} z_{k+1}
\end{array}\right]
$$

By the Guttman lemma $x_{k+1}^{T} p_{k+1} \neq 0$ holds if and only if

$$
\operatorname{rank}\left(x_{k+1}^{T} p_{k+1}\right)=\operatorname{rank}\left(\left(X^{\mid k+1}\right)^{T} H_{1}^{T}\left[Y^{\mid k}, z_{k+1}\right]\right)-\operatorname{rank}\left(X^{\mid k T} H_{1}^{T} Y^{\mid k}\right)=1 .
$$

This happens exactly if $\operatorname{rank}\left(\left(X^{\mid k+1}\right)^{T} H_{1}^{T}\left[Y^{\mid k}, z_{k+1}\right]\right)=k+1$, that is if

$$
\operatorname{det}\left(\left(X^{\mid k+1}\right)^{T} H_{1}^{T}\left[Y^{\mid k}, z_{k+1}\right]\right) \neq 0 \text {. }
$$

Thus we have

Theorem 23 Algorithm 2 produces a nonsingular $I$-conjugate pair $(P, X)$ if and only if the matrix $Y^{T} H_{1} X$ is strongly nonsingular, $x_{1}^{T} H_{1}^{T} z_{1} \neq 0$ and

$$
\operatorname{det}\left(\left(X^{\mid k+1}\right)^{T} H_{1}^{T}\left[Y^{\mid k}, z_{k+1}\right]\right) \neq 0 \quad(k=1, \ldots, m-1) .
$$

If we choose $z_{i}=y_{i}(i=1, \ldots, m)$, then condition (6.1) changes to

$$
x_{k}^{T} p_{k}=y_{k}^{T} H_{k} x_{k} \neq 0 \quad(i=1, \ldots, m) .
$$

Hence, the strong nonsingularity of $Y^{T} H_{1} X$ is the necessary and sufficient condition for producing nonsingular $I$-conjugate pairs $(X, P)$. The choice $Z=Y$ defines the generalized implicit $L U$ or $G I L U A B S$ subclass of the $A B S$ methods [13], [14]. Let us notice that $p_{i}=H_{i}^{T} y_{i}$ is the $i$ th component of $P_{m}$, the transpose of which we investigated in Section 5. As $P=P_{m}$ we can conclude that GILUABS conjugation is producing the pair $\left(X, P_{m}\right)$, which is $I$-conjugate. So the GILUABS conjugation is nothing else but a full rank factorization by Egerváry's rank reduction procedure. Similarly, we have that pair $\left(Q_{m}, Y\right)$ is also $I$-conjugate. Theorems 4 and 10 imply 
Theorem 24 Let $Z=Y$. Algorithm 2 produces a nonsingular I-conjugate pair $(P, X)$ if and only if the matrix $Y^{T} H_{1} X$ is strongly nonsingular. If $Y^{T} H_{1} X$ is strongly nonsingular, then $P=H_{1}^{T} Y L_{Y^{T} H_{1} X}^{-T}=H_{1}^{T} Y U_{X^{T} H_{1}^{T} Y}^{-1}$.

We prove the following characterizations of Algorithm 2.

Theorem 25 For any $I$-conjugate pair $(P, X)$ there exist an orthogonal matrix $Q$ and a nonsingular diagonal matrix $D$ such that Algorithm 2 with $H_{1}=I, Y=Z=Q D$ generates the pair $(P, X)$.

Proof. Let $P=Q D R_{1}$ be a $Q R$-factorization of $P$ with diagonal $D$ and unit upper triangular $R_{1}$. As $X^{T} P=X^{T} Q D R_{1}=L$ implies $X^{T} Q D=L R_{1}^{-1}$, the matrix $X^{T} Q D$ is strongly nonsingular. Hence by Theorem 24 Algorithm 2 produces exactly the pair $(P, X)$ for the parameters $H_{1}=I, Z=Y=Q D$.

For a given $X$ and orthogonal $Q$ there does not necessarily exist an $I$-conjugate pair $(P, X)$ produced by a $G I L U A B S$ conjugation. For example, let $H_{1}=I$,

$$
X=\left[\begin{array}{lll}
0 & 1 & 0 \\
1 & 1 & 0 \\
0 & 0 & 1
\end{array}\right], \quad Q=\left[\begin{array}{ccc}
1 & 0 & 0 \\
0 & \alpha & \beta \\
0 & -\beta & \alpha
\end{array}\right] \quad\left(\alpha^{2}+\beta^{2}=1\right)
$$

Then

$$
Y^{T} H_{1} X=Q^{T} X=\left[\begin{array}{ccc}
0 & 1 & 0 \\
\alpha & \alpha & -\beta \\
\beta & \beta & \alpha
\end{array}\right]
$$

is not strongly nonsingular.

Proposition 26 Let $Z=Y$. Algorithm 2 produces a nonsingular I-biconjugate pair $(P, X)$, if and only if the matrix $Y^{T} H_{1} X$ is lower triangular.

Proof. The pair $(P, X)$ is $I$-biconjugate, if $X^{T} P=D$ is diagonal. As $X^{T} P=$ $X^{T} H_{1}^{T} Y U_{X^{T} H_{1}^{T} Y}^{-1}=L_{X^{T} H_{1}^{T} Y} D_{X^{T} H_{1}^{T} Y}$ this may happen exactly if $L_{X^{T} H_{1}^{T} Y}$ is also diagonal.

Theorem 27 Let $B \in R^{m \times m}$ be a symmetric positive definite matrix and $Z=Y$. Algorithm 2 produces a nonsingular $I$-conjugate pair $(P, X)$ such that $P$ is $B$-orthogonal, if and only if $X=B H_{1}^{T} Y L^{T}$, where $Y$ is any nonsingular and $L$ is any nonsingular lower triangular matrix. In such a case $P=H_{1}^{T} Y U_{Y^{T} H_{1} B H_{1}^{T} Y}^{-1}$.

The same $P$ can be obtained, if we select $X=B P$ in Algorithm 2. Then $X^{T} P=$ $P^{T} B P=L$ implies that $L=D$ is diagonal,

$$
U_{X^{T} H_{1}^{T} Y}^{-T} Y^{T} H_{1} B H_{1}^{T} Y U_{X^{T} H_{1}^{T} Y}^{-1}=D,
$$


$Y^{T} H_{1} B H_{1}^{T} Y=U_{X^{T} H_{1}^{T} Y}^{T} D U_{X^{T} H_{1}^{T} Y}$ and $X=B H_{1}^{T} Y L^{T}$ with a nonsingular lower triangular $L$. In fact, the choice $X=B P=B H_{1}^{T} Y U_{X^{T} H_{1}^{T} Y}^{-1}$ corresponds to $L^{T}=$ $U_{X^{T} H_{1}^{T} Y}^{-1}$.

The above result appeared first in Stewart [25] for the special case $X=A^{T} V$, $V=A P, H_{1}=Z=Y=I$ and $L^{T}=I$, when $P=Y U_{Y^{T} A^{T} A Y}^{-1}$ (see also [3]).

\section{Stewart 's conjugation procedure}

Stewart's $A$-conjugation process is based on the $L U$ factorization $V^{T} A Q=L S$, where $Q=\left[q_{1}, \ldots, q_{m}\right]$ is the parameter [25]. Clearly, $\widetilde{P}=Q S^{-1}$ defines the $A$-conjugate pair $(\widetilde{P}, V)$. We prove that Stewart's $A$-conjugation procedure is equivalent with the GILUABS conjugation procedure. Hence it is also related to the rank reduction algorithm.

\section{STEWART'S CONJUGATION ALGORITHM}

$\tilde{p}_{1}=q_{1}$

for $k=1: m$

if $k>1$

$$
\begin{aligned}
s_{k} & =\left(L^{\mid \overline{k-1}}\right)^{-1}\left(V^{\mid k-1}\right)^{T} A q_{k} \\
\tilde{p}_{k} & =\sigma_{k k}^{-1}\left(q_{k}-\tilde{P}^{\mid k-1} s_{k}\right), \quad \sigma_{k k} \neq 0 \\
\text { end } & \\
L^{\mid \bar{k}} & =\left(V^{\mid k}\right)^{T} A \tilde{P}^{\mid k} \\
\text { end } &
\end{aligned}
$$

It is easy to see that

$$
\tilde{p}_{k+1}=\sigma_{k+1, k+1}^{-1}\left(I-\tilde{P}^{\mid k}\left(V^{\mid k T} A \tilde{P}^{\mid k}\right)^{-1} V^{\mid k T} A\right) q_{k+1} .
$$

As $\tilde{P}=Q S^{-1}$ we can write $\tilde{P}^{\mid k}=Q^{\mid k}\left(S^{-1}\right)^{\mid \bar{k}}$ and

$$
\tilde{P}^{\mid k}\left(V^{\mid k T} A \tilde{P}^{\mid k}\right)^{-1} V^{\mid k T} A=Q^{\mid k}\left\{V^{\mid k T} A Q^{\mid k}\right\}^{-1} V^{\mid k T} A .
$$

By the simple substitution $y_{k}=\sigma_{k k}^{-1} q_{k}$ we obtain that $\tilde{p}_{k}=H_{k}^{T} y_{k}$ for $k=1, \ldots, m$. Thus we proved that Stewart's conjugation procedure is algebraically equivalent with Algorithm 2 in the special case $Z=Y, H_{1}=I$ and $X=A^{T} V$. In fact, the Stewart algorithm implicitly uses the canonical form (3.3).

\section{The two-sided Gram-Schmidt method}

In this section we deal with the two-sided Gram-Schmidt (TSGS) process, which produces an $H_{1}$-biconjugate pair $(U, V)$. Let $H_{1} \in R^{m \times n}$ be of $\operatorname{rank} r, X=\left[x_{1}, \ldots, x_{r}\right]$ 
and $Y=\left[y_{1}, \ldots, y_{r}\right]$. The two-sided Gram-Schmidt algorithm is defined by

$$
\begin{aligned}
& u_{1}=x_{1}, v_{1}=y_{1} \\
& u_{k}=x_{k}-\sum_{i=1}^{k-1} \frac{v_{i}^{T} H_{1} x_{k}}{v_{i}^{T} H_{1} u_{i}} u_{i} \quad(k=2, \ldots, r) \\
& v_{k}=y_{k}-\sum_{i=1}^{k-1} \frac{y_{k}^{T} H_{1} u_{i}}{v_{i}^{T} H_{1} u_{i}} v_{i} \quad(k=2, \ldots, r)
\end{aligned}
$$

where $u_{k}$ and $v_{k}$ are the projections of $x_{k}$ and $y_{k}$, respectively.

This process was developed and rediscovered by several authors, the first of which is C. Hegedûs [16], [17],[18]. Hegedûs proved that the pair $(U, V)$ is $H_{1}$-biconjugate, $H_{1}=H_{1} U\left(V^{T} H_{1} U\right)^{-1} V^{T} H_{1}$, and $U\left(V^{T} H_{1} U\right)^{-1} V^{T}$ is a reflexive $g$-inverse of $H_{1}$ if the process is carried out. Parlett [21] defined algorithm (8.1)-(8.3) for $H_{1}=I \in$ $R^{m \times m}$. Chu, Funderlic and Golub [6] defined and investigated the TSGS biconjugation process in association with the Egerváry rank reduction algorithm. Finally we mention Broyden [5], who gave a block generalization of the Gram-Schmidt algorithm, which contains the TSGS biconjugation process of Hegedûs as a special case.

Using Theorem 10 we give a unique characterization of $U$ and $V$ in terms of the parameters $H_{1}, X$ and $Y$. We show that TSGS biconjugation is a special case of Egerváry's rank reduction algorithm.

Chu, Funderlic and Golub [6] proved the following result in association with the rank reduction algorithm $(2.2)$.

Theorem 28 (Chu-Funderlic-Golub [6]) Provided that process (8.1)-(8.3) is carried out, $H_{1} u_{k}=H_{k} x_{k}, v_{k}^{T} H_{1}=y_{k}^{T} H_{k}, y_{k}^{T} H_{k} x_{k}=v_{k}^{T} H_{1} u_{k}$ for all $k$ and $v_{i}^{T} H_{1} u_{j}=0$ for all $i \neq j$.

Let $U=\left[u_{1}, \ldots, u_{r}\right], V=\left[v_{1}, \ldots, v_{r}\right]$ and $\Omega=\operatorname{diag}\left(\omega_{i}\right)$. Then $V^{T} H_{1} U=\Omega$ is nonsingular diagonal by Theorem 28. Hence the pair $(U, V)$ is $H_{1}$-biconjugate. We can observe that $U$ and $V$ also satisfy the relations $Q_{r}=H_{1} U, \Omega=D_{r}$ and $P_{r}=H_{1}^{T} V$. Thus from the representation (5.3)-(5.4) we can assume that

$$
U=X U_{Y^{T} H_{1} X}^{-1}, \quad V=Y L_{Y^{T} H_{1} X}^{-T}=Y U_{X^{T} H_{1}^{T} Y}^{-1}
$$

Using the proof of Theorem 4 we can prove this and more.

Theorem 29 Let $H_{1} \in R^{m \times n}$ be of rank $r, X \in R^{n \times r}$ and $Y \in R^{m \times r}$ The TSGS biconjugation algorithm can be carried out if and only if Egerváry's rank reduction algorithm can be carried out. In such a case, when $Y^{T} H_{1} X$ is strongly nonsingular, relation (8.4) holds.

Proof. Let us assume first that $Y^{T} H_{1} X$ is strongly nonsingular. For $k=1$, $u_{1}=X U_{Y^{T} H_{1} X} e_{1}=X e_{1}=x_{1}$ and $v_{1}=Y L_{Y^{T} H_{1} X}^{-T} e_{1}=Y e_{1}=y_{1}$. Assuming the 
statement for $1 \leq i \leq k-1$ we can write

$$
v_{i}^{T} H_{1} u_{i}=e_{i}^{T} V^{T} H_{1} U e_{i}=e_{i}^{T} L_{Y^{T} H_{1} X}^{-1} Y^{T} H_{1} X U_{Y^{T} H_{1} X}^{-1} e_{i}=e_{i}^{T} D_{Y^{T} H_{1} X} e_{i}=\omega_{i} .
$$

By definition we have

$$
\begin{aligned}
u_{k} & =x_{k}-\sum_{i=1}^{k-1} \frac{v_{i}^{T} H_{1} x_{k}}{v_{i}^{T} H_{1} u_{i}} u_{i} \\
& =X e_{k}-\sum_{i=1}^{k-1} \frac{e_{i}^{T} L_{Y^{T} H_{1} X}^{-1} Y^{T} H_{1} X e_{k}}{\omega_{i}} X U_{Y^{T} H_{1} X}^{-1} e_{i} \\
& =X U_{Y^{T} H_{1} X}^{-1}\left(I-\sum_{i=1}^{k-1} \frac{e_{i} e_{i}^{T} D_{Y^{T} H_{1} X}}{\omega_{i}}\right) U_{Y^{T} H_{1} X} e_{k} \\
& =X U_{Y^{T} H_{1} X}^{-1} e_{k} .
\end{aligned}
$$

Similarly we have

$$
v_{k}=y_{k}-\sum_{i=1}^{k-1} \frac{y_{k}^{T} H_{1} u_{i}}{v_{i}^{T} H_{1} u_{i}} v_{i}=Y L_{Y^{T} H_{1} X}^{-T} e_{k}
$$

It is also clear that $v_{i}^{T} H_{1} u_{j}=e_{i}^{T} L_{Y^{T} H_{1} X}^{-1} Y^{T} H_{1} X U_{Y^{T} H_{1} X}^{-1} e_{j}=e_{i}^{T} D_{Y^{T} H_{1} X} e_{j}$ is zero, if $i \neq j$ and $\omega_{i}$ for $i=j(1 \leq i, j \leq k)$. Let us assume now that $Y^{T} H_{1} X$ is not strongly nonsingular. Then there is an index $t$ such that $y_{t}^{T} H_{t} x_{t}=0$ and $y_{i}^{T} H_{i} x_{i} \neq 0$ for $i<t$ (whenever $t>1$ ). Hence we can calculate $\left\{u_{i}, v_{i}\right\}_{i=1}^{t}$ with the TSGS algorithm. We cannot define, however, $u_{t+1}$ and $v_{t+1}$ because $y_{t}^{T} H_{t} x_{t}=v_{t}^{T} H_{1} u_{t}=0$. Thus we proved the theorem.

Let us notice that we also proved Theorem 28 .

The following part of the above theorem was formulated and proved in a different way by Chu, Funderlic and Golub [6].

Theorem 30 ([6], Thm. 2.2) Let $H_{1} \in R^{m \times n}$ be of rank $r, X \in R^{n \times r}$ and $Y \in$ $R^{m \times r}$. The TSGS biconjugation algorithm can be carried out, if and only if $Y^{T} H_{1} X$ is strongly nonsingular. In such a case, relation (8.4) holds.

This Theorem altogether with Theorem 4 also yields Theorem 29. The close relationship between the rank reduction and TSGS biconjugation is not at all surprising. Next we show that TSGS is a special case of Egerváry's rank reduction algorithm. We have two possibilities for doing this.

Let $H_{1}=A \in R^{m \times n}$ be of rank $r, X \in R^{n \times r}$ and $Y \in R^{m \times r}$ be such that $Y^{T} A X$ is strongly nonsingular. Let $A^{-}=X\left(Y^{T} A X\right)^{-1} Y^{T}$ be the reflexive $g$-inverse of $A$ 
produced implicitly by the rank reduction procedure (2.2). Let us define the rank reduction process

$$
\begin{aligned}
\widehat{H}_{1} & =A^{-} \\
\widehat{H}_{k+1} & =\widehat{H}_{k}-\widehat{H}_{k} A x_{k} y_{k}^{T} A \widehat{H}_{k} / y_{k}^{T} A \widehat{H}_{k} A x_{k} \quad(k=1, \ldots, r)
\end{aligned}
$$

and the related quantities

$$
\widehat{p}_{k}=\widehat{H}_{k}^{T} A^{T} y_{k}, \quad \widehat{q}_{k}=\widehat{H}_{k} A x_{k} \quad(k=1, \ldots, r) .
$$

As $\left(A^{T} Y\right)^{T} \widehat{H}_{1}(A X)=Y^{T} A A^{-} A X=Y^{T} A X$, the rank reduction process can be carried out, and by the special choice of $A^{-}$we have $\widehat{Q}_{r}=A^{-} A X U_{Y^{T} A X}^{-1}=X U_{Y^{T} A X}^{-1}$ and $\widehat{P}_{r}=\left(A^{-}\right)^{T} A^{T} Y L_{Y^{T} A X}^{-T}=Y L_{Y^{T}}^{-T} A X$. These are exactly $U$ and $V$ defined in (8.4). We have the following recursions

$$
\widehat{p}_{k+1}=\widehat{H}_{1}^{T} A^{T} y_{k+1}-\sum_{i=1}^{k} \frac{\widehat{q}_{i}^{T} A^{T} y_{k+1}}{y_{i}^{T} A \widehat{H}_{i} A x_{i}} \widehat{p}_{i}
$$

and

$$
\widehat{q}_{k+1}=\widehat{H}_{1} A x_{k+1}-\sum_{i=1}^{k} \frac{\widehat{p}_{i}^{T} A x_{k+1}}{y_{i}^{T} A \widehat{H}_{i} A x_{i}} \widehat{q}_{i} .
$$

As for reflexive $g$-inverses $\left(A^{-}\right)^{-}=A$, we can write

$$
y_{i}^{T} A \widehat{H}_{i} A x_{i}=y_{i}^{T} A \widehat{H}_{i} \widehat{H}_{1}^{-} \widehat{H}_{i} A x_{i}=\widehat{p}_{i}^{T} \widehat{H}_{1}^{-} \widehat{q}_{i}=\widehat{p}_{i}^{T} A \widehat{q}_{i} .
$$

Also, by the special choice of $A^{-}$, we have $\widehat{H}_{1}^{T} A^{T} y_{k+1}=y_{k+1}$ and $\widehat{H}_{1} A x_{k+1}=x_{k+1}$. Thus we have the following recursion

$$
\widehat{p}_{k+1}=y_{k+1}-\sum_{i=1}^{k} \frac{y_{k+1}^{T} A \widehat{q}_{i}}{\widehat{p}_{i}^{T} A \widehat{q}_{i}} \widehat{p}_{i}, \quad \widehat{q}_{k+1}=x_{k+1}-\sum_{i=1}^{k} \frac{\widehat{p}_{i}^{T} A x_{k+1}}{\widehat{p}_{i}^{T} A \widehat{q}_{i}} \widehat{q}_{i} .
$$

Letting $u_{k}=\widehat{q}_{k}$ and $v_{k}=\widehat{p}_{k}$ we obtain the TSGS recursion (8.1)-(8.3).

Theorem 31 The rank reduction process (8.5)-(8.7) can be written as TSGS biconjugation algorithm.

We can obtain the TSGS biconjugation process without using a special reflexive $g$-inverse, if we use two rank reduction processes as follows. Let $I_{m}$ denote the $m \times m$ unit matrix. Let

$$
\begin{aligned}
\widehat{H}_{1} & =I_{n} \\
\widehat{H}_{k+1} & =\widehat{H}_{k}-\widehat{H}_{k} A^{T} y_{k} x_{k}^{T} \widehat{H}_{k} / x_{k}^{T} \widehat{H}_{k} A^{T} y_{k} \quad(k=1, \ldots, r) \\
\widehat{p}_{k} & =\widehat{H}_{k}^{T} x_{k}
\end{aligned}
$$


and

$$
\begin{aligned}
H_{1} & =I_{m} \\
\widetilde{H}_{k+1} & =\widetilde{H}_{k}-\widetilde{H}_{k} A x_{k} y_{k}^{T} \widetilde{H}_{k} / y_{k}^{T} \widetilde{H}_{k} A x_{k} \quad(k=1, \ldots, r) \\
\widetilde{p}_{k} & =\widetilde{H}_{k}^{T} y_{k} .
\end{aligned}
$$

As $Y^{T} A X$ is strongly nonsingular, both recursions can be carried out. By Theorem $10 \widehat{P}_{r}=X L_{X^{T} A^{T} Y}^{-T}=X U_{Y^{T} A X}^{-1}$ and $\widetilde{P}_{r}=Y L_{Y^{T} A X}^{-T}$. These are exactly $U$ and $V$ defined by (8.4). Let us notice that the related quantities $\widehat{Q}_{r}$ and $\widetilde{Q}_{r}$ are not equal to $V$ or $U$. For $\widehat{P}_{r}$ and $\widetilde{P}_{r}$ we have the following recursions

$$
\widehat{p}_{k+1}=x_{k+1}-\sum_{i=1}^{k} \frac{y_{i}^{T} A \widehat{H}_{i}^{T} x_{k+1}}{x_{i}^{T} \widehat{H}_{i} A^{T} y_{i}} \widehat{p}_{i}
$$

and

$$
\widetilde{p}_{k+1}=y_{k+1}-\sum_{i=1}^{k} \frac{x_{i}^{T} A^{T} \widetilde{H}_{i}^{T} y_{k+1}}{y_{i}^{T} \widetilde{H}_{i} A x_{i}} \widetilde{p}_{i}
$$

Noticing that $A \widehat{H}_{i}^{T}=\widetilde{H}_{i} A$ we can write

$$
\begin{gathered}
y_{i}^{T} A \widehat{H}_{i}^{T} x_{k+1}=\widetilde{p}_{i}^{T} A x_{k+1}, \quad x_{i}^{T} A^{T} \widetilde{H}_{i}^{T} y_{k+1}=y_{k+1}^{T} A \widehat{p}_{i} \\
x_{i}^{T} \widehat{H}_{i} A^{T} y_{i}=y_{i}^{T} \widetilde{H}_{i} A x_{i}=y_{i}^{T} \widetilde{H}_{i} \widetilde{H}_{i} A x_{i}=y_{i}^{T} \widetilde{H}_{i} A \widehat{H}_{i}^{T} x_{i}=\widetilde{p}_{i}^{T} A \widehat{p}_{i} .
\end{gathered}
$$

By substitution we obtain the following recursion

$$
\widehat{p}_{k+1}=x_{k+1}-\sum_{i=1}^{k} \frac{\widetilde{p}_{i}^{T} A x_{k+1}}{\widetilde{p}_{i}^{T} A \widehat{p}_{i}} \widehat{p}_{i}, \quad \widetilde{p}_{k+1}=y_{k+1}-\sum_{i=1}^{k} \frac{y_{k+1}^{T} A \widehat{p}_{i}}{\widetilde{p}_{i}^{T} A \widehat{p}_{i}} \widetilde{p}_{i}
$$

Letting $u_{k}=\widehat{p}_{k}$ and $v_{k}=\widetilde{p}_{k}$ we obtain the TSGS biconjugation process.

Theorem 32 The two rank reduction procedures (8.9)-(8.11) and (8.12)-(8.14) can be written as TSGS algorithm.

Finally, we note that Abaffy and Spedicato proved that the TSGS algorithm of Hegedús (and also of Bodócs) is a special case of the ABS conjugation algorithm ([3], Thm. 8.30).

\section{Biconjugation and factorizations}

Although the TSGS biconjugation algorithm is a special case of the rank reduction algorithm, it is useful to review the following main results of Chu-Funderlic and Golub [6]. It is noted that we formulate and prove these results differently. 
Proposition 33 ([6], Thm. 3.2) Let $H_{1} \in R^{n \times n}$. For $X=Y=I$, then the TSGS algorithm can be carried out if and only if $H_{1}$ is strongly nonsingular. In this case the main diagonals of $A H_{1}, V^{T} H_{1}$, and $\Omega$ are identical and $H_{1}=V^{-T} \Omega U^{-1}$ is the unique LDU factorization of $H_{1}$.

Proof. The first part of the theorem follows from Theorem 29. If $X=I$ and $Y=I$, then $U=U_{H_{1}}^{-1}, V=L_{H_{1}}^{-T}$ and $\Omega=D_{H_{1}}$. Thus $H_{1} U=H_{1} U_{H_{1}}^{-1}=L_{H_{1}} D_{H_{1}}$, $V^{T} H_{1}=L_{H_{1}}^{-1} H_{1}=D_{H_{1}} U_{H_{1}}$ showing that all three matrices have the same diagonal. The product $V^{-T} \Omega U^{-1}=L_{H_{1}} D_{H_{1}} U_{H_{1}}$ is indeed an $L D U$ factorization of $H_{1}$.

Proposition 34 ([6], Thm. 3.3) If $H_{1} \in R^{n \times n}$ is symmetric and $X \in R^{n \times n}$ is such that $X^{T} H_{1} X$ is strongly nonsingular, then the resulting $H_{1}$-biconjugate pair $(U, V)$ has $U=V$. In this case, $V^{T} H_{1} U=\Omega$ is the canonical form of $H_{1}$ with respect to congruence and the columns of $U$ are A-orthogonal.

Proof. By Theorem $29 U=X U_{X^{T} H_{1} X}^{-1}$ and $V=X L_{X^{T} H_{1} X}^{-T}=X U_{X^{T} H_{1} X}^{-1}$. Thus $U=V$ and

$$
V^{T} H_{1} U=V^{T} H_{1} V=L_{X^{T} H_{1} X}^{-1} X^{T} H_{1} X U_{X^{T} H_{1} X}=D_{X^{T} H_{1} X} .
$$

Proposition 35 ([6], Thm. 3.6) Let $H_{1} \in R^{m \times n}$ have full column rank, $X=I$ and $Y=H_{1}$. Then the TSGS biconjugation can be carried out and the resulting $H_{1}$-biconjugate pair $(U, V)$ is such that $Q=V D_{H_{1}^{T} H_{1}}^{-1 / 2}$ and $R=D_{H_{1}^{T} H_{1}}^{1 / 2} U$ defines a QR-factorization of $H_{1}$.

Proof. The $Y^{T} H_{1} X=H_{1}^{T} H_{1}$ is symmetric and positive definite. Hence $U=$ $U_{H_{1}^{T} H_{1}}^{-1}, V=H_{1} U_{H_{1}^{T} H_{1}}^{-1}$. It is clear that $Q=H_{1} U_{H_{1}^{T} H_{1}}^{-1} D_{H_{1}^{T} H_{1}}^{-1 / 2}$ is orthogonal, and $Q$ and $R=D_{H_{1}^{T} H_{1}}^{1 / 2} U_{H_{1}^{T} H_{1}}$ define the $Q R$-factorization of $H_{1}$.

For quadratic matrices this result appears in several forms (see, e.g. Stewart [25], Abaffy-Spedicato [3] and Section 5 of this paper).

Proposition 36 ([6], Thm. 3.7) Let $H_{1} \in R^{m \times n}$ have the singular value decomposition $H_{1}=Y \Sigma X^{T}$, where $Y^{T} Y=I=X^{T} X$ and $\Sigma \in R^{r \times r}$ is nonsingular. For these $X$ and $Y$ the TSGS biconjugation algorithm produces the $H_{1}$-biconjugate pair $(X, Y)$.

Proof. By definition $Y^{T} H_{1} X=\Sigma$ is strongly nonsingular. So the biconjugation can be carried out. Also by definition $U=X U_{Y^{T} H_{1} X}^{-1}=X$ and $V=Y L_{Y^{T} H_{1} X}^{-T}=Y$.

This result is a special case of Proposition 12.

Proposition 37 ([6], Thm. 3.8) Let the nonzero singular values of $H_{1}$ be ordered such that $\omega_{1} \geq \omega_{2} \geq \ldots \geq \omega_{r}>0$. If in the rank reducing process $\omega_{k+1}=\left\|H_{k+1}\right\|_{2}$, 
then the corresponding $x_{k+1}$ and $y_{k+1}$ vectors may be chosen to be the corresponding singular vectors of $H_{1}$, and the largest singular value of $H_{k+1}$ is the $(k+1)$ th largest singular value of $H_{1}$.

The result is a special case of Proposition 6 with the choice $F=X$ and $G=\Sigma X^{T}$.

\section{Acknowledgments}

This paper was written to celebrate the 75th birthday of Prof. Pál Rózsa of the Technical University of Budapest. The author is expressing his gratitude to Prof. Pál Rózsa for his help concerning Egerváry's work in linear algebra. A useful discussion with Prof. C. Broyden is acknowledged. Profs. Abaffy J., Hegedûs C. and E. Spedicato also made helpful comments on the manuscript.

\section{REFERENCES}

[1] Abaffy J., Broyden, C.G., Spedichto, E.: A class of direct methods for linear equations, Numerische Mathematik, 45, (1984), 361-376.

[2] Abaffy J., Galántai A.: Conjugate direction methods for linear and nonlinear systems of algebraic equations, Colloquia Mathematica Soc. János Bolyai, 50. Numerical Methods, Miskolc (Hungary) 1986. (ed. P. Rózsa) North-Holland, Amsterdam, 1987, 481-502.

[3] Abaffy J., Spedichto, E.: ABS-projection Algorithms: Mathematical Techniques for Linear and Nonlinear Algebraic Equations, Ellis Horwood, Chichester, 1989.

[4] Broyden, C.G.: On the numerical stability of Huang's and related methods, JOTA, 47, (1985), 401-412.

[5] Broyden, C.G.: The Gram-Schmidt method - a hierarchy of algorithms, in: A. Sydow, editor, 15th IMACS World Congress on Scientific Computation, Modelling and Applied Mathematics, Vol. 2, Numerical Mathematics, Wissenschaft and Technik Verlag, Berlin 1997, 545-550.

[6] Chu, M.T., Funderlic, R.E., Golub, G.H.: A rank-one reduction formula and its applications to matrix factorizations, SIAM Review, 37, (1995), 512-530.

[7] Cline, R.E., Funderlic, R.E.: The rank of a difference of matrices and associated generalized inverses, Lin. Alg. Appl., 24, (1979), 185-215.

[8] Egerváry J.: Über die Faktorisation von Matrizen und ihre Anwendung auf die Lösung von linearen Gleichungssystemen, Zeitschrift für angewandte Mathematik und Mechanik 35, (1955), 111-118.

[9] Egerváry, J.: Az inverz mátrix általánosítása (On a generalized inverse for matrices, in Hungarian), A Magyar Tudományos Akadémia Matematikai Kutató Intézetének Közleményei (Publications of the Mathematical Institute of the Hungarian Academy of Sciences), 1(3), (1956), 315-324.

[10] Egerváry, E.: On rank-diminishing operations and their applications to the solution of linear equations, ZAMP, XI., (1960), 376-386.

[11] Egervány J.: Über eine Methode zur numerischen Lösung der Poissonschen Differen- 
zengleichung für beliebige Gebiete, Acta Mathematica Academiae Scientiarium Hungaricae 11, (1960), 341-361.

[12] Elsner, L., Rózsa P.: On eigenvectors and adjoints of modified matrices, Linear and Multilinear Algebra, 10, (1981), 235-247.

[13] Galántai A.: Generalized implicit LU algorithms in the class of ABS methods for linear and nonlinear systems of algebraic equations, Quaderno DMSIA 93/5, University of Bergamo, Bergamo, 1993.

[14] Galántai A.: The global convergence of the ABS methods for a class of nonlinear problems, Optimization Methods and Software, 4, (1995) 283-295.

[15] Guttman, L.: Enlargement methods for computing the inverse matrix, Ann. Math. Statist. 17, (1946), 336-343.

[16] Hegedûs C.J.: Generalization of the method of conjugate gradients: The method of conjugate pairs, in: Collection of Scientific Papers in Collaboration with the Joint Institute for Nuclear Research, Dubna, USSR and the Central Research Institute for Physics, Budapest, Hungary, Algorithms and Programs for Solution of Some Problems in Physics (Report KFKI 1979-82, MTA KFKI, Budapest, 1979, 199-209.

[17] Hegedûs C.J., Bodócs L.: Konjugált irányok előállítása: a konjugált párok módszere, (Generation of conjugate directions: The method of conjugate pairs, in Hungarian), Alkalmazott Matematikai Lapok, 11, (1985), 297-309.

[18] Hegedûs C.J.: Generating conjugate directions for arbitrary matrices by matrix equations, I-II., Computers Math. Applic. 21, (1991), 71-94.

[19] Householder, A.S.: The Theory of Matrices in Numerical Analysis, Blaisdell, New York, 1964.

[20] Ouellette, D.V.: Schur complements and statistics, Lin. Alg. Appl., 36, (1981), 187-295.

[21] Parlett, B.N.: Reduction to tridiagonal form and minimal realizations, SIAM J. Matrix Anal. Appl., 13, (1992), 567-593.

[22] RAO, C.R.: Linear Statistical Inference and Its Applications, Wiley, New York, 1965.

[23] Rogge, T.R., Walling, D.D.: A note on the bordering method of inverting a matrix, Am. Math. Monthly, 73, (1966), 879-890.

[24] Rózsa P.: Lineáris algebra és alkalmazásai (Linear algebra and its applications, in Hungarian), Müszaki Könyvkiadó, Budapest, 1974.

[25] Stewart, G.W.: Conjugate direction methods for solving systems of linear equations, Num. Math., 21, (1973), 285-297.

[26] Voyevodin, V.V.: Linear algebra, Mir, Moscow, 1983.

[27] Watkins, D.S., Elsner, L.: Convergence of algorithms of decomposition type for the eigenvalue problem, Linear Algebra Appl., 143, (1991), 19-47.

[28] Wedderburn, J.H.M.: Lectures on Matrices, Amer. Math. Soc. Colloquium Publications, Vol. XVII., 1934. 